Iramar Baptistella do Nascimento OChtps://orcid. org/0000-0003-1268-2777

Cristiano Rech Bitencourt ${ }^{2}$ Ohtps://orid.org/0000-0002-7814-9142

Raquel Fleig ${ }^{3}$

Ohttps://orcid.org/0000-0003-1934-6936

\section{Estratégias para o transtorno do espectro autista: interação social e intervenções terapêuticas}

\author{
Strategies for autism spectrum disorder: social interaction \\ and therapeutic interventions
}

D0l: $10.1590 / 0047-2085000000326$

\begin{abstract}
RESUMO
Objetivo: Identificar os fatores que dificultam as intervenções terapêuticas motoras em crianças com transtorno do espectro autista. Métodos: Foram utilizadas as bases de dados PubMed, Web of Science, Scopus e LILACS. Uma pesquisa de literatura cinzenta foi conduzida com acadêmico do Google. PRISMA foi usado, bem como a análise de risco de viés adaptada do Cochrane Manual para ensaios clínicos e, para outros estudos, foi utilizada a lista de verificação Downs e Black. Resultados: Dezessete artigos científicos foram incluídos na análise de síntese qualitativa sobre estratégias para interação social e motora em pacientes com transtorno do espectro autista. Quatorze artigos científicos alcançaram pontuações aceitáveis na escala de Downs e Black, e três ensaios clínicos indicaram domínios satisfatórios. Pessoas com transtorno do espectro autista apresentaram percentuais acima de 30\%, com dificuldades de comportamentos sociáveis, de aprendizagem e de comunicação. O diagnóstico adequado e a preconização de um plano de tratamento para o desenvolvimento da motricidade são estratégias fundamentais e de ordem prioritária, uma vez que possibilitarão uma análise quantitativa ao longo da vida do autista, assim como proporcionarão uma maior viabilidade de análise das habilidades cognitivas. Conclusão: A dificuldade de comportamento social do indivíduo com transtorno do espectro autista pode ser o ponto-alvo nas suas limitações de aprendizagem motora. Portanto, a concepção metodológica com a prática de imitação, associada a uma dinâmica prazerosa do exercício rítmico, é a melhor sugestão para contemplar o direcionamento das pesquisas contemporâneas, uma vez que, quando prevalece a inter-relação entre interação social, aprendizagem motora e percepções sensoriais, os desfechos são mais significativos para essas crianças.
\end{abstract}

\section{PALAVRAS-CHAVE}

Transtorno do espectro autista, adaptação psicológica, terapia de modalidade combinada.

\section{ABSTRACT}

Objective: To identify the factors that hinder motor therapeutic interventions in children with autism spectrum disorder. Methods: The PubMed, Web of Science, Scopus, and LILACS databases were used. A Grey Literature search was conducted with a Google scholar. PRISMA was used, as well as the risk analysis of bias adapted from the Cochrane Manual for clinical trials and, for other studies, the Downs and Black checklist was used. Results: Seventeen scientific articles were included in the qualitative synthesis analysis on strategies for social and motor interaction in patients with autism spectrum disorder. Fourteen scientific articles achieved acceptable scores on the Downs and Black scale, and three clinical trials indicated satisfactory domains. People with autism spectrum disorder showed percentages above $30 \%$, with difficulties in sociable behavior, learning, and communication issues. Adequate diagnosis and advocacy of a treatment plan for the development of motor skills are fundamental and priority strategies since they will enable a quantitative analysis throughout the life of the autistic person, as well as providing greater viability for the analysis of cognitive skills. Conclusion: The difficulty of social behavior of the individual with autism spectrum disorder may be the target point in his motor learning limitations. Therefore, the methodological conception with the practice of imitation, associated with a pleasurable dynamic of rhythmic exercise, is the best suggestion to contemplate the direction of contemporary research, since when the interrelation between social interaction, motor learning, and sensory perceptions prevails, outcomes are more significant for these children.

\section{KEYWORDS}

Autism spectrum disorder, psychological adaptation, combined mode therapy.

Received in: 0ct/2/2020. Approved in: Feb/17/2021

1 Universidade do Estado de Santa Catarina, Departamento de Ciências da Saúde, Florianópolis, SC, Brasil.

2 Universidade do Estado de Santa Catarina, Centro de Ciências da Saúde e do Esporte, Programa de Pós-graduação em Ciência do Movimento Humano, Florianópolis, SC, Brasil.

3 Universidade do Estado de Santa Catarina, Florianópolis, SC, Brasil.

Address for correspondence: Iramar Baptistella do Nascimento. Rua Pascoal Simone, 358, Coqueiros - 88080-350 - Florianópolis, SC, Brasil. E-mail: iramar.nascimento@udesc.br 


\section{INTRODUÇÃO}

A ciência vem estudando o autismo há muitos anos, visto que os conceitos sobre o distúrbio dessa alteração ainda se encontram alicerçados pelo Manual Diagnóstico e Estatístico de Transtornos Mentais, o DSM-5 (2014)'. O transtorno do espectro autista (TEA) manifesta-se desde as idades mais precoces, frequentemente antes dos 3 anos até a fase adulta, dependendo dos graus de autismo ${ }^{1,2}$. Vale acrescentar também que cerca de $75 \%$ dos autistas demonstram deficiência mental e $1 \%$ da população mundial é diagnosticada com TEA ${ }^{2-4}$

Entre os fatores que podem impactar a atuação dos profissionais da saúde, estão aspectos físicos e educacionais e a perda de controle motor seletivo, que é característico de uma criança autista ${ }^{5}$. Outra questão relevante diz respeito às adversidades do autista no âmbito social e à baixa qualidade de vida de seus familiares decorrente das atribuições de suas vidas cotidianas ${ }^{6-9}$

Dessa forma, a atuação profissional exige não apenas compreender a presença de subsequentes comorbidades, mas também os fatores sintomáticos e idiopáticos que resultam em uma maior complexidade para o profissional de saúde entender a capacidade do autista em estabelecer relações ${ }^{9}$. Já se tornou notório, na literatura científica, a ansiedade das mães em relação a uma terapia que favoreça e modifique o comportamento de seus filhos, amenizando os sintomas e a baixa qualidade de vida de ambos ${ }^{7,9,10}$. No entanto, os grupos categóricos relacionados ao autismo divergem quanto a transtornos que mostram incapacidade de relacionamento com o seu próximo, pacientes com aversão ao toque e ainda, em alguns casos, autistas que apresentam um forte apego aos pais, intensificando os níveis de estresse ${ }^{6,10}$.

Nas condições inerentes às disfunções motoras, 50\% dos indivíduos com TEA apresentam dificuldades motoras, incluindo descoordenação em atividades motoras finas, desajeitado padrão de marcha e capacidade de equilíbrio reduzida $^{11,12}$. Todavia, as alterações no trato motor relacionadas com o seu desenvolvimento podem ser detectadas antes das desordens de aspectos sociais e anormalidades linguísticas. Portanto, o presente estudo teve como propósito identificar os fatores que dificultam as intervenções terapêuticas motoras em crianças com TEA.

\section{MÉTODOS}

Uma revisão sistemática da literatura foi desenvolvida. No decorrer desta pesquisa, foram utilizadas as diretrizes de acordo com a lista de verificação Itens Preferidos para Revisões Sistemáticas e Metanálises (PRISMA) ${ }^{13}$. Seguindo as diretrizes do protocolo preestabelecido, as características metodológicas dos estudos foram coletadas da seguinte forma: a busca foi efetuada por meio de dados provenientes de fontes primárias ou secundárias contidos nos estudos de ensaios clínicos, estudos de coorte prospectivos, retrospectivos, casos controle e estudos transversais. Para esta pesquisa, também foram considerados estudos de revisão, epidemiológicos, estudos de estratégia de buscas na literatura para revisões sistemáticas e livros. Vale ressaltar, no entanto, que a análise desses estudos serviu apenas para enriquecer a presente pesquisa, mas as conclusões e dados daí obtidos não foram computados na análise qualitativa. Também não foram considerados artigos pessoais, editoriais, cartas, resenhas, comentários e resumos de congressos.

$\mathrm{Na}$ pesquisa literária, foi aplicada a estratégia $\mathrm{PICO}^{14}$. População: pacientes crianças com autismo, submetidos à monitoração sobre suas relações, comportamentos e tratamentos. Intervenção: terapias visando a uma melhora nos aspectos físicos, educacionais e maior controle motor seletivo. Controle: estudos sobre diferentes estratégias utilizadas e a identificação de comparativos entre os grupos de autistas. Resultados: hipotetizou-se a identificação de variáveis que possibilitará melhores diretrizes para os tratamentos terapêuticos no paciente autista. O presente estudo buscou uma concordância homogênea das características específicas das amostras dos pacientes.

\section{Fontes de informação e desenvolvimento das estratégias de busca}

A busca foi realizada nas bases de dados Web of Science, Scopus, PubMed/MEDLINE e Literatura Latino-Americana e do Caribe em Ciências da Saúde (LILACS). Para a pesquisa de 50 primeiros artigos da literatura cinza, foi utilizado o banco de dados do Google Scholar. Após a obtenção dos estudos incluídos, foi desenvolvida uma revisão manual para fornecer uma avaliação mais precisa. Para a obtenção das palavras-chave, verificou-se nos descritores em ciências da saúde (DeCS) da LILACS, uma Biblioteca Virtual em Saúde. A busca foi realizada nos meses de abril e maio de 2020. Os descritores "autism spectrum disorder", "combined modality therapy" e "adaptation, psychological" foram associados aos operadores boleanos "AND" e "OR".

Consequentemente, um processo de seleção referencial foi aplicado para revisões sistemáticas seguindo estas etapas: identificação por meio dos locais de pesquisa mencionados acima; triagem de títulos e resumos; elegibilidade da população; métodos, relevância do projeto; dados de associação sobre as terapêuticas com o autista e resultados; estudos avaliando fatores que influenciam nas dificuldades de tratamentos; parâmetros antropométricos e funcionalidade do paciente e como ele se manifesta; bem como os resultados e validade da estratégia aplicada. Dois autores extraíram os dados que apresentavam maior relevância nos respectivos sites.

Subsequentemente, a análise foi desenvolvida de forma independente e, se houvesse discórdia, os dois autores 
deveriam pedir o parecer de um terceiro revisor, que deveria revisar e seguir as prioridades estabelecidas no protocolo inicial, priorizando o ano mais recente, a metodologia com escopo mais amplo e a força de evidência científica. Vale ressaltar que, se qualquer informação não ficasse bem esclarecida por causa da falta de dados ou por qualquer outro motivo, os autores dos artigos seriam contatados para esclarecimentos.

Extração dos dados: os autores tiveram o objetivo de coletar e organizar dados e, de forma descritiva no texto, identificaram os países onde os estudos de seleção qualitativa foram realizados. Outra proposta, para facilitar a interpretação do leitor, foi o desenvolvimento de uma tabela sobre as características metodológicas dos estudos, com as estatísticas e os equipamentos relacionados e com o uso de diferentes tipos de estudos clínicos.

Critérios de inclusão: as pesquisas poderiam ser efetuadas apenas em estudos com crianças contendo o diagnóstico de autismo, ou seja, pesquisas que abordassem técnicas e estratégias para a criança com TEA e avaliação durante o seu desenvolvimento.

Critério de exclusão: artigos científicos não relevantes que abordaram outras condições e que não fossem focados nos fatores que pudessem impactar resultados e estratégias para o desenvolvimento da motricidade e a melhora da interatividade social durante o tratamento com a criança autista. Também foram excluídos artigos com outras populações.

\section{Principais temas avaliados}

- Estratégias para a interação social em pacientes com TEA

- Interação motora em pacientes com TEA

\section{Avaliação do risco de viés em estudos selecionados}

Os estudos de ensaios clínicos seguiram as diretrizes do Cochrane Handbook for Systematic Reviews of Interventions (Versão 5.1.0) $)^{15}$. Desenvolveu-se uma adaptação da ferramenta para verificação de viés na tabela 8.5.d (Manual Cochrane de Revisões Sistemáticas de Intervenções, versão 5.1.0, diretrizes). Os pesquisadores avaliaram e consideraram os resultados da seguinte maneira: foram considerados satisfatórios e de possível alocação, quando um determinado estudo atingiu " $\geq 4$ " domínios da tabela com baixo nível de viés. Um estudo para ser selecionado deveria apresentar baixo risco de viés, preferencialmente nos domínios seis e sete, ou seja, superioridade no baixo nível de viés em quatro domínios ou mais, uma vez que contemplou o sexto e sétimo domínios. Foi considerado insatisfatório para este estudo quando um estudo atingiu "baixo risco de viés" em apenas um, dois ou três domínios " $\leq 3$ ".

Já em outros estudos como os de coorte, caso-controle e corte transversal, o nível de viés foi avaliado por uma adaptação das escalas de Downs e Black ${ }^{16}$. Essa escala tem como objetivo avaliar estudos não relacionados a ensaios clínicos, e sim estudos de coorte, caso-controle e corte transversal. Para esses estudos, a pontuação foi alocada da seguinte forma: para uma pesquisa ser selecionada, ela deveria atingir 13 pontos, pelo menos, independentemente do tipo de estudo. No entanto, a pontuação máxima para os estudos de caso-controle foi estipulada em 28 pontos de acordo com os critérios da escala e 22 pontos no máximo, tanto para os estudos de coorte quanto para os de corte transversal.

\section{RESULTADOS}

\section{Seleção dos estudos}

Utilizando os bancos de dados selecionados para procurar os artigos, 361 artigos foram identificados sobre o tema de interesse. Depois da remoção de 83 artigos duplicados, 278 artigos em língua portuguesa, inglês e espanhol foram obtidos para a análise. Uma análise abrangente do título e do resumo eliminou 237 artigos, resultando em 41 artigos na primeira etapa do o estudo. Com base na análise dos 50 primeiros resultados do Google Scholar, dois novos artigos foram adicionados da literatura cinzenta e, subsequentemente, três artigos foram incluídos a partir das referências dos estudos selecionados, totalizando 46 artigos elegíveis para a segunda etapa da revisão. Na segunda etapa, todos os 46 artigos foram lidos na íntegra e 29 foram excluídos da análise - 13, pela falta de dados para identificar estratégias utilizadas para a interação social e motora da criança com autismo; sete porque avaliaram outros desfechos; oito porque forneceram dados insuficientes para avaliar as relações entre terapeuta, criança autista e intercorrências; um porque a população avaliada apresentava outras doenças que se somaram aos desfechos do autismo com a possibilidade de viés. O fluxograma mostrando o processo de identificação, inclusão e exclusão de estudos é mais bem detalhado na figura 1.

\section{Características gerais da pesquisa relacionadas ao tipo de estudo e ao país desenvolvido}

A presente revisão sistemática, após a aplicação da seleção condizente com os critérios acima mencionados, obteve, na inclusão, 17 estudos científicos para a análise qualitativa, dos quais seis $(35,3 \%)$ de coorte prospectiva, cinco $(29,4 \%)$ de coorte retrospectiva, três $(17,6 \%)$ de ensaio clínico, dois $(11,7 \%)$ de corte transversal e um $(5,9 \%)$ de caso-controle. Quanto ao país onde se desenvolveu o estudo, o quadro é o seguinte: sete $(41,2 \%)$ foram desenvolvidos nos Estados Unidos, seis $(35,3 \%)$ no Brasil, um $(5,6 \%)$ na Alemanha, um $(5,6 \%)$ no Irã, um $(5,6 \%)$ na China e um $(5,6 \%)$ na Irlanda do Norte (Tabelas 1 e 2). 


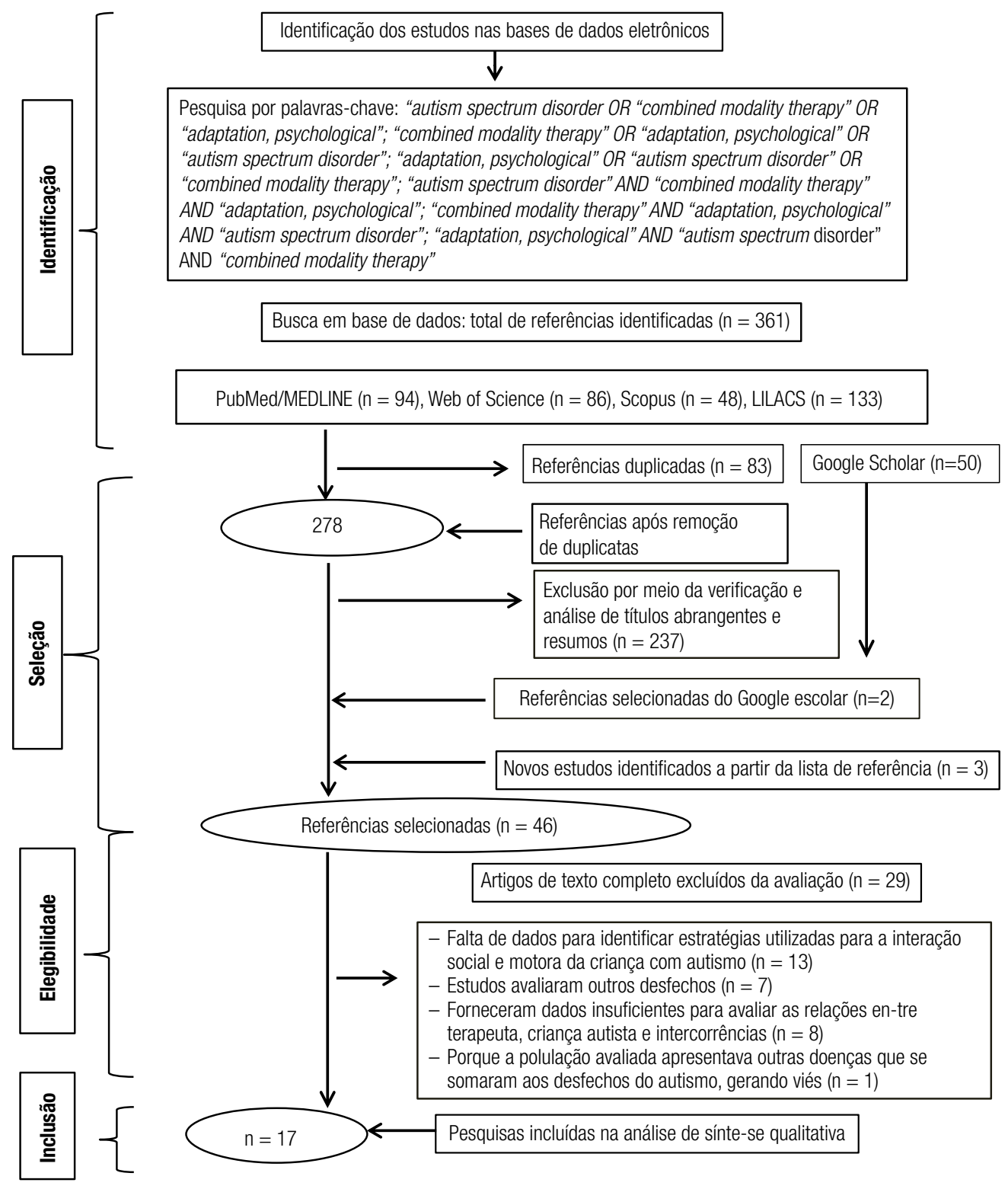

Figura 1. Fluxograma das atividades do processo de seleção por meio do diagrama de busca bibliográfica adaptado cheklist PRISMA.

\section{Soma absoluta dos dados em relação ao número de pesquisas e pontuação alcançadas}

Em relação às pontuações da escala de Downs e Black adaptada, dois estudos alcançaram 17 pontos, quatro estudos alcançaram 16 pontos e seis atingiram 13 pontos (Tabela 3). Com adaptação da ferramenta de verificação de viés do Manual Cochrane, um estudo de ensaio clínico teve baixo risco de viés em cinco (domínios, que foi a maior proporção de baixo risco de viés, e dois alcançaram baixo nível de viés em quatro domínios (Tabela 4).

\section{Síntese dos resultados}

Os estudos identificaram os principais fatores que dificultam a interação social da criança com TEA. Concomitantemente, os estudos têm demonstrado que existe uma dificuldade de desenvolver amostras homogêneas para o desenvolvimento de ensaios clínicos com pacientes autistas, provavelmente por causa de suas dificuldades de interação. Vale ressaltar que a falta de estudos de intervenção com crianças com autismo é muito grande na literatura científica, e as atividades rítmicas e as estratégias que envolvem uma dinâmica de imitação no processo educativo de aprendizagem parecem ser um conteúdo interessante a ser explorado nessa população. Verificou-se que pais com idade mais avançada são mais propensos a ter filhos com esse transtorno.

Parece existir uma relação entre o comprometimento na aprendizagem motora e a dificuldade de interação social. Essa é uma afirmativa consistente, visto que as pessoas com TEA apresentaram percentuais acima de 30\% para dificuldades relativas a comportamentos sociáveis, aprendizagem 
Tabela 1. Características dos estudos observacionais incluídos na síntese qualitativa e pontuações para a escala de Downs e Black adaptada

\begin{tabular}{|c|c|c|c|c|c|}
\hline Autores & País e ano & $\begin{array}{l}\text { Número de } \\
\text { participantes }\end{array}$ & $\begin{array}{l}\text { Tipo de } \\
\text { estudo }\end{array}$ & População de análise & Principais resultados \\
\hline $\begin{array}{l}\text { Zablotsky } \\
\text { et al. }\end{array}$ & $\begin{array}{l}\text { Estados Unidos } \\
2012\end{array}$ & $n=1.110$ & $\mathrm{CR}$ & $\begin{array}{l}\text { Crianças com TEA, a partir de } 4 \text { anos, } \\
\text { chegando a adultos de } 18 \text { anos }\end{array}$ & $\begin{array}{l}\text { Autismo e transtornos psiquiátricos estão associados à } \\
\text { depressão materna e baixa qualidade de vida. }\end{array}$ \\
\hline $\begin{array}{l}\text { Misquiatti } \\
\text { et al. }\end{array}$ & $\begin{array}{l}\text { Brasil } \\
2015\end{array}$ & $\mathrm{n}=20$ & CT & Crianças com TEA, de 3 a 10 anos & $\begin{array}{l}\text { Crianças com transtornos do espectro do autismo podem } \\
\text { sobrecarregar seus familiares. }\end{array}$ \\
\hline $\begin{array}{l}\text { Yeargin-Allsopp } \\
\text { et al. }{ }^{10}\end{array}$ & $\begin{array}{l}\text { Estados Unidos } \\
2003\end{array}$ & $n=987$ & $\mathrm{CR}$ & Crianças com TEA, de 3 a 10 anos & $\begin{array}{l}\text { A prevalência de autismo na proporção homem-mulher foi de } \\
4: 1 \text {. }\end{array}$ \\
\hline Hilton et al. ${ }^{11}$ & $\begin{array}{l}\text { Estados Unidos } \\
2012\end{array}$ & $n=144$ & CR & $\begin{array}{l}\text { Crianças com TEA, a partir de } 4 \text { anos, } \\
\text { chegando a adultos de } 21 \text { anos }\end{array}$ & $\begin{array}{l}\text { As habilidades motoras prejudicadas estão associadas, entre as } \\
\text { crianças afetadas por TEA, à gravidade do autismo e ao Ql. }\end{array}$ \\
\hline Maia et al. ${ }^{18}$ & $\begin{array}{l}\text { Brasil } \\
2018\end{array}$ & $n=253$ & CC & $\begin{array}{l}\text { Crianças com TEA, a partir de } 2 \text { anos, } \\
\text { chegando à adolescência }\end{array}$ & $\begin{array}{l}\text { A idade dos pais no parto indicou uma forte associação com } \\
\text { TEA. É necessário enfatizar a prevenção para o parto tardio e } \\
\text { acompanhamento das crianças nascidas desses casais. }\end{array}$ \\
\hline Miccas et al..20 & $\begin{array}{l}\text { Brasil } \\
2014\end{array}$ & $n=12$ & $\mathrm{CP}$ & $\begin{array}{l}\text { Crianças com TEA, a partir de } 8 \text { anos } \\
\text { chegando a adultos de } 22 \text { anos }\end{array}$ & $\begin{array}{l}\text { Existem diferenças significativas entre idade, ano/série e pontuação } \\
\text { segundo a avaliação de funcionalidade. São fundamentais a } \\
\text { discussão e o planejamento no ambiente escolar. }\end{array}$ \\
\hline Ingersol|23 & $\begin{array}{l}\text { Estados Unidos } \\
2008\end{array}$ & $n=14$ & $\mathrm{CP}$ & Crianças com TEA de 2 a 5 anos & $\begin{array}{l}\text { Crianças com autismo imitaram menos do que crianças com } \\
\text { desenvolvimento típico em geral. }\end{array}$ \\
\hline Koch et al. ${ }^{27}$ & $\begin{array}{l}\text { Alemanha } \\
2015\end{array}$ & $\mathrm{n}=31$ & $\mathrm{CP}$ & Crianças com TEA até 6 anos & $\begin{array}{l}\text { A terapia do movimento de dança pode ser uma abordagem } \\
\text { eficaz e viável para o transtorno do TEA. }\end{array}$ \\
\hline Memari et al. ${ }^{29}$ & $\begin{array}{l}\text { Irã } \\
2012\end{array}$ & $\mathrm{n}=80$ & $\mathrm{CP}$ & Crianças e adolescentes com TEA & $\begin{array}{l}\text { Estrutura familiar, atividades sedentárias, comorbidades e } \\
\text { obesidade foram identificadas como outros determinantes de } \\
\text { padrões de atividade física em crianças com TEA. }\end{array}$ \\
\hline Yu et al. ${ }^{33}$ & $\begin{array}{l}\text { China } \\
2018\end{array}$ & $n=127$ & $\mathrm{CR}$ & 30 a 71 meses & $\begin{array}{l}\text { A terapêutica com crianças em idade pré-escolar com TEA que } \\
\text { estão desenvolvendo habilidades motoras finas ou realizando } \\
\text { tarefas motoras finas relacionadas à coordenação visomotora } \\
\text { deve reforçar a atenção ao QI das crianças. }\end{array}$ \\
\hline Kaur et al. ${ }^{34}$ & $\begin{array}{l}\text { Estados Unidos } \\
2018\end{array}$ & $n=24$ & $\mathrm{CP}$ & Crianças com TEA de 5 a 12 anos & $\begin{array}{l}\text { Tanto o desempenho motor fino quanto o grosso se } \\
\text { correlacionaram significativamente com o Ql, mas não com a } \\
\text { gravidade do autismo; no entanto, os erros de práxis } \\
\text { (principalmente, total, estouro e ritmicidade) estão fortemente } \\
\text { correlacionados com a gravidade do autismo, e não com o QI. } \\
\text { Médicos e terapeutas devem incluir avaliações motoras e } \\
\text { intervenções no padrão de atendimento de crianças com TEA. }\end{array}$ \\
\hline Rocha et al..$^{36}$ & $\begin{array}{l}\text { Brasil } \\
2020\end{array}$ & $n=685$ & $\mathrm{CR}$ & Crianças com TEA de 0 a 12 anos & $\begin{array}{l}\text { Existe a necessidade de instrumentos qualificados para avaliação } \\
\text { do diagnóstico e capacitação continuada para os diferentes } \\
\text { profissionais que atuam com crianças com TEA. }\end{array}$ \\
\hline Garcia et al..$^{38}$ & $\begin{array}{l}\text { Brasil } \\
2016\end{array}$ & $n=151$ & CT & Busca de diagnóstico de TEA & $\begin{array}{l}\text { Protocolos clínicos básicos e disponíveis em nosso meio são } \\
\text { eficazes tanto para validar o diagnóstico como para conhecer } \\
\text { melhor o perfil cognitivo-comportamental dos alunos com autismo. }\end{array}$ \\
\hline $\begin{array}{l}\text { McPhillips } \\
\text { et al. } .^{39}\end{array}$ & $\begin{array}{l}\text { Irlanda do } \\
\text { Norte }\end{array}$ & $\mathrm{n}=147$ & $\mathrm{CP}$ & $\begin{array}{l}\text { Crianças com TEA, entre } 3 \text { a } 10 \\
\text { meses }\end{array}$ & $\begin{array}{l}\text { Crianças com TEA e DEL correm o risco de déficits motores } \\
\text { clinicamente significativos. No entanto, futuros estudos } \\
\text { comportamentais e neurológicos de habilidades motoras em } \\
\text { crianças com TEA devem incluir um grupo de comparação com } \\
\text { DEL, a fim de identificar possíveis déficits específicos do autismo. }\end{array}$ \\
\hline
\end{tabular}

n: número de indivíduos da amostra; SO: score obtido; PM: pontuação máxima; CR: coorte retrospectiva; CP: coorte prospectiva; CT: corte transversal; TEA: transtorno do espectro autista; Ql: quociente de inteligência, DEL: distúrbio específico de linguagem.

Tabela 2. Características dos estudos incluídos na síntese qualitativa dos ensaios clínicos

\begin{tabular}{|c|c|c|c|c|}
\hline Autores & País e ano & $\begin{array}{c}\text { Número de } \\
\text { participantes }\end{array}$ & $\begin{array}{l}\text { Tipo de } \\
\text { estudo }\end{array}$ & Resultados \\
\hline Ingersol|122 & $\begin{array}{l}\text { Estados Unidos } \\
2010\end{array}$ & $n=21$ & EC & $\begin{array}{l}0 \text { número de brincadeiras espontâneas no pré-tratamento foi relacionado a melhorias na imitação } \\
\text { durante a intervenção, sugerindo que crianças com um repertório lúdico maior obtêm maiores ganhos } \\
\text { durante a RIT. }\end{array}$ \\
\hline Krüger et al. ${ }^{24}$ & $\begin{array}{l}\text { Brasil } \\
2019\end{array}$ & $\mathrm{n}=10$ & EC & $\begin{array}{l}\text { Quatorze semanas de atividades rítmicas podem ser uma ferramenta eficaz para desenvolver as } \\
\text { habilidades motoras de crianças com transtorno do espectro autista. }\end{array}$ \\
\hline MacDonald et al. ${ }^{28}$ & $\begin{array}{l}\text { Estados Unidos } \\
2011\end{array}$ & $\mathrm{n}=72$ & EC & $\begin{array}{l}\text { Crianças mais velhas com transtorno do espectro do autismo são significativamente mais inativas } \\
\text { fisicamente, em comparação com crianças mais novas. Programas e intervenções de atividade física } \\
\text { precisam abordar esse déficit, na atividade física. }\end{array}$ \\
\hline
\end{tabular}


Tabela 3. Estudos observacionais incluídos na síntese qualitativa e pontuações para a escala adaptada de Downs e Black

\begin{tabular}{lcc}
\hline Autores & $\begin{array}{c}\text { Downs e Black } \\
\text { SO/MS }\end{array}$ & $\begin{array}{c}\text { Frequência relativa } \\
\text { (\%) }\end{array}$ \\
\hline Zablotsky et al. ${ }^{6}$ & $13 / 22$ & 59,1 \\
Misquiatti et al. ${ }^{8}$ & $16 / 22$ & 72,7 \\
Yeargin-Allsopp et al. ${ }^{10}$ & $16 / 22$ & 72,7 \\
Hilton et al. ${ }^{11}$ & $17 / 22$ & 77,3 \\
Maia et al. ${ }^{18}$ & $20 / 28$ & 71,4 \\
Miccas et al.. & $13 / 22$ & 59,1 \\
Ingersoll.23 & $13 / 22$ & 59,1 \\
Koch et al. ${ }^{27}$ & $16 / 22$ & 72,7 \\
Memari et al. ${ }^{29}$ & $13 / 22$ & 59,1 \\
Yu et al. ${ }^{33}$ & $13 / 22$ & 59,1 \\
Kaur et al. ${ }^{34}$ & $16 / 22$ & 72,7 \\
Rocha et al. ${ }^{36}$ & $13 / 22$ & 59,1 \\
Garcia et al. ${ }^{38}$ & $16 / 22$ & 72,7 \\
McPhillips et al. & $17 / 22$ & 77,3 \\
\hline
\end{tabular}

SO: score obtido; MS: pontuação máxima.

Tabela 4. Estudos de ensaios clínicos e o número de domínios encontrados a partir da adaptação da ferramenta de verificação de viés do Manual Cochrane

\begin{tabular}{lcc}
\hline Autores & $\begin{array}{c}\text { Cochrane Handbook } \\
\text { DO/MD }\end{array}$ & $\begin{array}{c}\text { Frequência relativa } \\
\text { (\%) }\end{array}$ \\
\hline Ingersoll22 & $4 / 7$ & 57,1 \\
Krüger et al. ${ }^{24}$ & $5 / 7$ & 71,4 \\
MacDonald et al.. & $4 / 7$ & 57,1 \\
\hline
\end{tabular}

DO: domínio encontrado; MD: número máximo de domínios.

e comunicação. Um protocolo padronizado com medidas quantitativas é imprescindível para análise quantitativa da motricidade. Dessa forma, o diagnóstico adequado e a preconização de um plano de tratamento para o desenvolvimento da motricidade são estratégias fundamentais e de ordem prioritária, uma vez que possibilitarão uma análise quantitativa ao longo da vida do autista, assim como poderão favorecer a análise das habilidades cognitivas.

\section{DISCUSSÃO}

\section{Estratégias para interação social em pacientes com TEA}

Melhorar as dificuldades de interação social do paciente com TEA é um dos obstáculos a ser conquistado ${ }^{17}$. O TEA tornou-se um problema de saúde pública, com forte impacto social, econômico e familiar. A idade reprodutiva tardia é um fator relevante para o nascimento de crianças com esse transtorno, e o rastreamento e acompanhamentos delas crianças devem ser enfatizados, tendo em vista a razão de chances (OR) de 2,87 quando pelo menos um dos genitores apresentavam idade avançada (pai $\geq 45$ e mãe $\geq 35$ ), (OR: 2,87; intervalo de confiança [IC] de 95\%: 1,56-5,26) e de 4,22 quando ambos tinham idades avançadas (OR: 4,22; IC de 95\%: 1,63-10,95) ${ }^{18}$. Em uma comparativa entre sexos, a prevalência de autismo foi de 3,4 por 1.000 (IC de 95\%: 3,2-3,6) - proporção homem-mulher: 4:110.

As tradições das pesquisas científicas com TEA são de grupos limitados em suas amostras, impossibilitando, muitas vezes, a identificação e a análise de resultados compatíveis que favoreçam novas metodologias ${ }^{17}$. Estudos mostram as dificuldades de desenvolver estratégias e coletar dados nos projetos de políticas de sistemas educacionais em maior escala para a escolarização de crianças e jovens com autismo ${ }^{17,19}$.

O Programa de Atenção aos Transtornos do Espectro (PAED) é utilizado para demonstrar a funcionalidade de indivíduos com TEA por meio de protocolos elaborados e sistematizados na proposta de Classificação Internacional de Funcionalidade (CIF) com o propósito de avaliar questões referentes à Classificação Internacional de Doenças (CID), caracterizando-se a importância de um questionário confiável para avaliar os alunos com TEA 20,21 . Uma pesquisa do ano de 2014, numa escola especial de ensino fundamental com o uso do PAED, teve o objetivo de identificar a avaliação funcional no domínio de atividades e participação dos alunos com TEA. Os resultados apresentaram alunos com dificuldades nos seguintes itens: $48 \%$ da amostra não realizava o conhecimento aprendido, 32\% não apresentaram um comportamento sociável e uma média de 31,4\% dos pacientes tinha dificuldades de comunicação ${ }^{20}$.

Outra estratégia que demonstrou relevância nas interações sociais para crianças com TEA foi desenvolvida em um estudo de ensaio clínico randomizado com um método de Treinamento de Imitação Recíproca (RIT) aplicado em crianças autistas $^{22}$. A mesma proposta metodológica já havia sido desenvolvida em um estudo precedente com crianças com TEA, e os resultados foram semelhantes quanto às dificuldades na sua capacidade de imitar em contextos sociais espontâneos e aos prognósticos desfavoráveis ${ }^{23}$. No entanto, no estudo mais recente ${ }^{22}$, os estudiosos evidenciaram que os ganhos a curto prazo nas habilidades de imitação eram perceptíveis e, consecutivamente, recomendaram a utilização do RIT a longo prazo, uma vez que os seus efeitos sugerem um impacto positivo no ganho de linguagem e principalmente no desenvolvimento social com o uso dessa técnica ${ }^{22}$.

Outros autores hipotetizaram a ideia de que as atividades rítmicas seriam um método interessante a ser tratado nessa população, visto que se enquadram em uma estratégia de baixo custo e a dinâmica possibilita uma expressão corporal de acordo com as necessidades do momento ${ }^{24}$. A proposta 
pressupõe a aquisição de novos fundamentos que poderiam subsidiar pesquisas contemporâneas. No entanto, desfechos em uma amostra contendo crianças com TEA, com o propósito de identificar as atividades rítmicas nas interações sociais por meio do efeito estandardizado mediante o uso do método "d de Cohen"25, apontaram efeitos não significativos com as atividades rítmicas, e os resultados foram semelhantes no momento pré-teste ( $p=0,556)$ e não houve diferença para interação social após as 14 semanas de intervenção $(p=0,111)^{24}$.

Todavia, sabe-se que os benefícios das atividades rítmicas podem melhorar o desenvolvimento social e estabelece-se, durante a sua execução, um ambiente agradável e de fácil aprendizagem ${ }^{26}$. Trata-se de uma contextualização consistente, uma vez que estudos científicos anteriores com indivíduos com TEA apresentaram melhorias nas habilidades sociais ${ }^{27}$. Em contraposição, mesmo que a prática de exercícios com atividades rítmicas se sustente em concepções relacionadas a aspectos positivos de tratamento e a qualidade de vida do paciente, diferentes pesquisas destacam que indivíduos com TEA apresentam níveis reduzidos na execução de atividades motoras ${ }^{28,29}$.

As anormalidades qualitativas e quantitativas atingem não apenas as áreas de interação social, mas também a comunicação e as áreas de comportamento ${ }^{30}$. Portanto, o reconhecimento da variabilidade do grau de comprometimento do indivíduo com TEA tornou-se importante para o processo avaliativo. No entanto, existe a necessidade de desenvolver ou validar instrumentos de avaliação diagnóstica para o sexo feminino, e as diferenças entre os sexos podem comprometer a eficiência diagnóstica ${ }^{31}$. Assim sendo, esses comentários destacam qualquer interpretação generalizada quanto aos desfechos referente às suas habilidades ${ }^{30,31}$.

\section{Interação motora em pacientes com TEA}

A aprendizagem motora da criança se estabelece por meio de um processo que se desenvolve por uma interação constante e permanente do indivíduo com o meio familiar e escolar ${ }^{32}$. A estratégia de desenvolver uma análise antecipada das habilidades motoras finas no ambiente pré-escolar de indivíduos com TEA parece ser fundamental para ampliar a motricidade, visto que permite identificar antecipadamente o déficit de coordenação e percepção da motricidade ${ }^{33}$.

Nos indivíduos com TEA, fortes evidências apontam transtornos do desenvolvimento da coordenação (TDC), tanto para o comprometimento das habilidades motoras grossas, incluindo a coordenação bilateral, quanto para a motricidade fina, referente à habilidade de caligrafia ${ }^{34,35}$. Já sobre prognósticos futuros, a deficiência motora na infância parece ser um preconizador para o comprometimento da criança com TEA, principalmente nas áreas sociais e cognitivas e na sua comunicação. Logo, o profissional fisioterapeuta é de total importância no desenvolvimento da psicomotricidade, evolução na estabilidade de equilíbrio e coordenação motora, destacando-se a necessidade de instrumentos qualificados para a avaliação do diagnóstico ${ }^{36,37}$.

Nos achados científicos, dois aspectos se apresentaram de forma relevante para conhecer melhor o perfil cognitivo-comportamental e condições gerais de saúde dos alunos com autismo: o registro clínico com observações minuciosas e o perfil da equipe multidisciplinar, que deverá conhecer a etiologia e a heterogeneidade clínica do TEA ${ }^{38}$. Entre as relações referentes às percepções sensoriais e motoras que existem em crianças com TEA, pode-se constatar a mútua dificuldade de estímulos sensoriais e motores decorrentes de desordens voluntárias e involuntárias ${ }^{31}$.

Determinadas habilidade como corrida, corrida lateral, laterais alternados e salto horizontal são exemplos de atividades que podem ser fundamentais, embora já se tenha conhecimento sobre os limites da pessoa com TEA ${ }^{39}$. O diagnóstico adequado e um plano de tratamento motor são fatores que podem disponibilizar uma perspectiva inovadora para as áreas de cognição, comunicação social e comportamento, ainda que o diagnóstico de TEA necessite de uma triagem motora adequada, com medidas motoras válidas e estendendo-se a intervenções e avaliações de profissionais especializados e/ou de terapeutas físicos que abordem as deficiências funcionais ${ }^{40}$.

Outros pesquisadores ratificaram a importância da avaliação padronizada da função motora e sugerem que os testes sejam validados em crianças com TEA. Tal diretriz é importante, dada à heterogeneidade dessa particularidade clíni$\mathrm{ca}^{41}$. Além disso, a aplicabilidade de medidas quantitativas da função motora oportunizará análises comparativas desses indivíduos ao longo da vida útil, com habilidades comportamentais cognitivas e variadas.

Vale destacar que a utilização do PAED foi relevante em alguns resultados de compreensão por meio dos sentidos, e a estratégia somou-se aos resultados de desfechos de motricidade. Uma amostra com 12 alunos com TEA apontou a área com menos dificuldade de percepção sensorial, com 69,2\% que realizaram versus (vs.) 30,8\% que não realizam a percepção sensitiva, e a área de coordenação motora, com 59,6\% vs. 40,4\%, respectivamente ${ }^{20}$. Sobre a coordenação motora, o estudo de Krüger et al. ${ }^{24}$ avaliou o efeito da intervenção baseada em exercícios físicos nas habilidades motoras no pré e pós-testes, para os grupos intervenção e controle. Em crianças com TEA, o estudo apresentou melhora nas habilidades motoras após 14 semanas de atividades rítmicas, com $p=$ 0,042; tamanho do efeito de 1,86 (d de Cohen). Já no grupo controle, não apontou diferença significativa $(p=0,317)$. $O$ estudo concluiu que 14 semanas de atividades rítmicas pode ser um método eficaz para desenvolver as habilidades motoras em pessoas com TEA. 


\section{CONCLUSÃO}

A restrição de estudos mais recentes com intervenção na área motora envolvendo indivíduos com TEA é relevante. A dificuldade de comportamento social do indivíduo com TEA pode ser o ponto-alvo nas suas limitações de aprendizagem motora. O diagnóstico alinhado e um consecutivo plano de tratamento priorizando o desenvolvimento motor são de fundamental importância. Portanto, a concepção metodológica com a prática de imitação, associada a uma dinâmica prazerosa do exercício rítmico, é a melhor sugestão para contemplar o direcionamento das pesquisas contemporâneas, uma vez que, quando prevalece a inter-relação entre interação social, aprendizagem motora e percepções sensoriais, os desfechos são mais significativos para essas crianças.

\section{CONTRIBUIÇÃO DOS AUTORES}

Todos os autores participaram ativamente de todas as etapas de elaboração do manuscrito: (1) contribuíram significativamente na concepção e desenho dos estudos ou na análise e interpretação dos dados; (2) contribuíram substancialmente na elaboração do artigo ou revisando criticamente o seu conteúdo intelectual e (3) aprovaram sua versão final a ser publicada.

\section{COMITÊ DE ÉTICA}

O manuscrito não envolve pesquisa com seres humanos, justificando a não aprovação por Comitê de Ética.

\section{CONFLITO DE INTERESSES}

Nenhum.

\section{AGRADECIMENTOS}

Não houve fonte de financiamento.

\section{REFERÊNCIAS}

1. Lamb JA, Moore J, Bailey AMA. Autism: recent molecular genetic advances. Hum Mol Genet. 2000;9(6):1-22.

2. Smalley SL. Genetic influences in childhood-onset psychiatric disorders: autism and attention-deficit/hyperactivity disorder. Am J Hum Genet. 1997;60(6):1276-82.

3. Do LLTN. American Psychiatric Association Diagnostic and Statistical Manual of Mental Disorders (DSM-IV). Encycl Child Behav Dev. 2011;84-5. Disponível em: https://scigraph. springernature.com/pub.10.1007/978-0-387-79061-9_113. Acesso em: 11 jan. 2020.
4. Organização Mundial de Saúde (OMS). Classificação estatística internacional de doenças e problemas relacionados à saúde: (ID-10. 10a revisão. São Paulo: OMS; 2000. p. 361-2. Disponível em: https://www.edusp.com.br/livros/cid-10-1. Acesso em: 13 jan. 2020.

5. Scazufca M. Brazilian version of the Burden Interview scale for the assessment of burden of care in carers of people with mental illnesses. Rev Bras Psiquiatr. 2002;24(1):12-7.

6. Zablotsky B, Anderson CLP. The association between child autism symptomatology, maternal quality of life, and risk for depression. J Autism Dev Disord. 2013;26(4):1-37.

7. Gardiner E, larocci G. Unhappy (and happy) in their own way: a developmental psychopathology perspective on quality of life for families living with developmental disability with and without autism. Res Dev Disabil. 2012;33(6):2177-92.

8. Misquiatti ARN, Brito MC, Ferreira FTS, Assumpção Júnior FB. Sobrecarga familiar e crianças com transtornos do espectro do autismo: perspectiva dos cuidadores. Rev CEFAC. 2015;17(1):192-200.

9. Chaim MPM, Neto SBC, Pereira AYF, Costa VESM. Phenomenology of the quality of life of autistic children. Rev Abordagem Gestalt. 2020;26(2):122-34

10. Yeargin-Allsopp M, Rice C, Karapurkar T, Doemberg N, Boyle C, Murphy C. Prevalence of autism in a US metropolitan area. JAMA. 2003;289(1):49-55.

11. Hilton CL, Zhang Y, Whilte MR, Klohr CL, Constantino J. Motor impairment in sibling pairs concordant and discordant for autism spectrum disorders. 2012;16(4):430-41.

12. Bhat AN, Landa RJ, Galloway JC. Current perspectives on motor functioning in infants, children, and adults with autism spectrum disorders. Phys Ther. 2011;91(7):1116-29.

13. Galvão TF, Pansani TSA, Harrad D. Principais itens para relatar Revisões sistemáticas e Metanálises: A recomendação PRISMA. Epidemiol Serv Saúde. 2015;24(2).

14. Santos CMC, Pimenta CAM, Nobre MRC. A estratégia PICO para a construção da pergunta de pesquisa e busca de evidências. Ver Latino-Am Enfermagem. 2007;15(3):508-11.

15. Higgins JP, Green S. Cochrane Handbook for Systematic Reviews of Interventios. Version 5.1.0. 2011. Disponível em: https://training.cochrane.org/handbook. Acesso em: 13 jan. 2020.

16. Downs SH, Black N. The feasibility of creating a checklist for the assessment of the methodological quality both of randomized and non-randomised studies of health care interventions. J Epidemiol Community Health. 1998;52(6):377-84

17. Gomes CGS, Mendes EG. Escolarização inclusiva de alunos com autismo na rede municipal de ensino de Belo Horizonte. Rev Bras Educ Espec. 2010;16(3):375-96.

18. Maia FA, Almeida MTC, Alves MR, Bandeira LVS, Silva VB, Nunes NF, et al. Autism spectrum disorder and parents' age: A case-control study in Brazil. Cad Saúde Pública. 2018:34(8):e00109917.

19. Hallmayer J, Cleveland BS, Torres AMA. Genetic heritability and shared environmental factors among twin pairs with autism. Arch Gen Psychiatry. 2011;68(11):1095-102.

20. Miccas C, Vital AAF, D’Antino MEF. Avaliação de funcionalidade em atividades e participação de alunos com transtornos do espectro do autismo. Rev Psicopedag. 2014;31(94):3-10.

21. Caravale B, Baldi S, Gasparini C, Wilson BN. Cross-Cultural adaptation, reliability and predictive validity of the Italian version of Developmental Coordination Disorder Questionnaire (DCDQ). Eur J Paediatr Neurol. 2014;18(3):267-72.

22. Ingersoll B. Brief report: Pilot randomized controlled trial of reciprocal imitation training for teaching elicited and spontaneous imitation to children with autism. J Autism Dev Disord. 2010;40(9):1154-60.

23. Ingersoll B. The effect of context on imitation skills in children with autism. Res Autism Spectr Disord. 2008;2(2):332-40.

24. Krüger GR, Garcias LM, Hax GP, Marques AC. 0 efeito de um programa de atividades rítmicas na interação social e na coordenação motora em crianças com transtorno do espectro autista. Rev Bras Ativ Fís Saúde. 2019;23(1):1-5.

25. Cohen J. Statistical power analysis for the behavioral sciences. 2nd ed. Hillsdale, NJ: Lawrence Earlbaum Associates; 1988.

26. Campeiz ECFS, Volpi CM. Dança criativa: a qualidade da experiência subjetiva. Motriz. 2004;10(3):167-72

27. Koch SC, Mehl L, Sobanski E, Sieber MFT, Fuchs T. Fixing the mirrors: a feasibility study of the effects of dance movement therapy on young adults with autism spectrum disorder. Autism. 2015;19(3):338-50. 
28. Macdonald M, Esposito P, Ulrich D. The physical activity patterns of children with autism. BMC Res Notes. 2011;4(422):1-5.

29. Memari AH, Ghaheri B, Ziaee V, Kordi R, Hafizi S, Moshayedi P. Physical activity in children and adolescents with autism assessed by triaxial accelerometry. Pediatr 0 bes. 2013:8(2):150-8.

30. Schwartzman JS, Araújo CA, orgs. Transtornos do espectro do autismo - TEA. São Paulo: Memnon; 2011.

31. Evêncio KMM, Menezes HCS, Fernandes GP. Transtorno do espectro do autismo: considerações sobre o diagnóstico. Id on Line Rev. 2019;13(47):234-51.

32. Weiss MLL. Psicopedagogia clínica: uma visão diagnóstica dos problemas de aprendizagem escolar. 14a ed. rev. e ampl. Rio de Janeiro: Lamparina; 2012.

33. Yu TY, Chou W, Chow JC, Lin CH, Tung LC, Chen KL. IQ discrepancy differentiates levels of fine motor skills and their relationship in children with autism spectrum disorders. Neuropsychiatr Dis Treat. 2018;14(1):597-605.

34. Kaur M, Srinivasan SM, Bhat AN. Comparing motor performance, praxis, coordination, and interpersonal synchrony between children with and without Autism Spectrum Disorder (ASD). Res Dev Disabil. 2018;72(1):79-95.
35. Fleury A, Kushki A, Tanel N, Anagnostou E, Chau T. Statistical persistence and timing characteristics of repetitive circle drawing in children with ASD. Dev Neurorehabil. 2013;16(4):245-54.

36. Rocha CC, Souza SMV, Costa AF, Portes JRM. 0 perfil da população infantil com suspeita de diagnóstico de transtorno do espectro autista atendida por um Centro Especializado em Reabilitação de uma cidade do Sul do Brasil. Physis. 2019;29(4):1-20.

37. Oliveira EM, Gonçalves FTD, Magalhães MM, Nascimento HMS, Carvalho ICV, Lemos AVL. 0 impacto da psicomotricidade no tratamento de crianças com transtorno do espectro autista: revisão integrativa. Revista Eletrônica Acervo Saúde. 2019;34(1):e1369.

38. Garcia AHC, Viveiros MM, Schwartzman JS, Brunoni D. Transtornos do Espectro do Autismo: Avaliação e Comorbidades em Alunos de Barueri, São Paulo. Psicol Teor Prat. 2016;18(1):166-77.

39. McPhillips M, Finlay J, Bejerot S, Hanley M. Motor deficits in children with autism spectrum disorder: a cross-syndrome study. Autism Res. 2014;7(6):664-76.

40. Bhat AN. Is Motor Impairment in Autism Spectrum Disorder Distinct From Developmental Coordination Disorder? A Report From the SPARK Study. Phys Ther. 2020;100(4):633-44.

41. Wilson RB, MCCracken JT, Rinehart NJ, Jeste SS. What's missing in autism spectrum disorder motor assessments? J Neurodevelop Disord. 2013;10(33):1-13. 